\title{
El modelo de patrones de aprendizaje: estado actual, reflexiones y perspectivas desde el territorio de Iberoamérica
}

Learning Pattern Model: Current State, Reflections and Perspectives from Ibero-American Territory

O modelo de padrões de aprendizagem: status atual, reflexões e perspectivas do território da Ibero-América

Jose Reinaldo Martínez-Fernández* iD orcid.org/0000-0002-1233-7386

\section{(c) $\underset{\mathrm{BY}}{(1)}$}

Recibido: 17/05/2019

Evaluado: 24/05/2019

Doctor en Psicología Cognitiva. Profesor Agregado. Departamento de Psicología Evolutiva y de la Educación, Universitat Autònoma de Barcelona, España. 


\section{Resumen}

Este artículo de revisión y reflexión se planteó con el objetivo de presentar el modelo teórico e instrumental referido a la identificación y el fomento de los patrones de aprendizaje (Vermunt, 1998): un modelo integrador de los componentes de creencias, motivaciones y estrategias en el análisis de los procesos de aprendizaje. Pero, además, un modelo gestado con una clara visión del papel de ciertos factores contextuales y personales en la discusión de sus vínculos direccionales y la configuración propia de los patrones (Vermunt, 2005). En términos científicos, se trata de un modelo reciente que ha tenido sendas revisiones metaanalíticas lideradas por su autor (Vermunt y Donche, 2017; Vermunt y Vermetten, 2004). Aquí, en lo que concierne a la revisión, se retoman tales contribuciones para describir el modelo y su historia; se elabora una breve presentación de su utilización en el territorio iberoamericano para conocer el estado actual en cuanto a los patrones de aprendizaje en esta parte del mundo; y se hace una revisión final de las potencialidades, debilidades y perspectivas con relación a los patrones de aprendizaje. En conjunto, se analiza el rol que tienen los ambientes de aprendizaje, los conocimientos previos, la edad, el género y la dimensión cultural para entender por qué prevalecen ciertas formas de aprender, de crecer, de concebir y actuar con la información.

\section{Palabras clave}

patrones de aprendizaje; procesos de aprendizaje; estrategias de aprendizaje; patrones socioculturales; estudios transculturales

\section{Keywords}

learning patterns; learning processes; learning strategies; sociocultural patterns; crosscultural studies

\begin{abstract}
The purpose of this review and reflection article was to present the theoretical and instrumental model to identify and promote learning patterns (Vermunt, 1998). This model integrates beliefs, motivation and strategies in the analysis of learning processes. It arose with a clear conception of the role of background and personal factors in the discussion of its goals, and the configuration of the patterns (Vermunt, 2005). Scientifically speaking, it is a recent model that has been revised by Vermunt and some of his colleagues (Vermunt \& Donche, 2017; Vermunt \& Vermetten, 2004). In this review, the authors go back to those contributions and describe the model and its history. After a brief presentation of its use in Ibero-America, they present a state of the art of learning patterns in this region. Finally, they discuss the potentialities, weaknesses and perspectives of learning patterns. All in all, there is an analysis of the role played by learning environments, previous knowledge, age, gender, and culture to understand the prevalence of some ways to learn, grow, conceive, and act with information.
\end{abstract}

\section{Resumo}

Este artigo de revisão e reflexão foi produzido com o objetivo de apresentar o modelo teórico e instrumental referente à identificação e promoção de padrões de aprendizagem (Vermunt, 1998): um modelo integrador dos componentes das crenças, motivações e estratégias no análise de processos de aprendizagem. Mas, além disso, um modelo gestado com uma visão clara do papel de certos fatores contextuais e pessoais na discussão de seus links direcionais e na configuração dos próprios padrões (Vermunt, 2005). Em termos científicos, é um modelo recente que teve revisões metaanalíticas lideradas por seu autor (Vermunt e Donche, 2017, Vermunt e Vermetten, 2004). Aqui, no que diz respeito à revisão, tais contribuições são retomadas para descrever o modelo e sua história; uma breve apresentação de seu uso é elaborada no território ibero-americano para conhecer o estado atual em relação aos padrões de aprendizagem nesta parte do mundo; e uma revisão final dos potenciais, fraquezas e perspectivas em relação aos padrões de aprendizagem é feita. Como um todo, o papel dos ambientes de aprendizagem, o conhecimento prévio, a idade, o gênero e a dimensão cultural são analisados para entender por que certas formas de aprender, de crescer, de conceber e agir com a informação prevalecem.

\section{Palavras-chave}

Padrões de aprendizagem; processos de aprendizagem; estratégias de aprendizagem; padrões socioculturais; estudos transculturais 


\section{Introducción}

El estudio del modelo de patrones de aprendizaje, en Iberoamérica, se inicia a mediados de la primera década del siglo xxı con las aportaciones de Alves de Lima et al. (2006) y Vázquez (2009) desde Argentina. Sin embargo, es a partir del 2009 cuando un grupo de investigadores liderados por Martínez-Fernández revisa, adapta y traduce el cuestionario ILS para abordar el análisis de los patrones de aprendizaje, a la Vermunt, en dicho territorio (Martínez-Fernández et al. 2009). Desde entonces, los datos reportados por muestras de estudiantes universitarios de Colombia, España, México y Venezuela, confirman la identificación de los patrones típicamente universales, como el MD, el AD, el RD y el UD. Sin embargo, también se identifican ciertos matices que responden a particularidades contextuales, o quizás evolutivas, y que remiten a otro tipo de composición entre creencias y acciones, como los patrones pasivo-idealista, pasivo-motivacional, flexible, o combinaciones entre los componentes que quizás se expliquen por la relación con ciertos momentos evolutivos de cambio o ajuste, y por ello se trata de patrones asociados con estudiantes de menor edad (Vermunt y Vermetten, 2004). Sin embargo, hoy en día parece que esa menor coherencia entre los elementos que definen los patrones se debe más a una serie de influencias contextuales y culturales que a una cuestión evolutiva (García-Ravidá, 2017; Martínez-Fernández y Vermunt, 2015; Vermunt, et al., 2014). Aquí es importante destacar uno de los aspectos que Vermunt (2005) ya menciona como influyentes en la configuración de los patrones de aprendizaje: los factores evolutivos de las personas y los factores contextuales enraizados en la dimensión cultural.

En suma, se trata de un modelo que ha tenido cierta repercusión en el mundo de la psicología de la educación, pero es poco lo que se ha abordado de manera metaanalítica e integrada a partir de la información de estudiantes provenientes de diferentes países de lberoamérica (una aproximación es la de Martínez-Fernández y Vermunt, 2015). De este modo, aún queda camino por recorrer en cuanto a la discusión en profundidad del modelo de los patrones de aprendizaje y su influencia en los procesos de aprendizaje desde una visión más internacional e inclusiva de las ciencias del comportamiento y de la educación. Es decir, la construcción de un modelo teórico e instrumental con un mayor abanico de interpretaciones que se puedan generar de los procesos de aprendizaje, y sobre todo de las orientaciones para fomentar el mejoramiento constante en dichos procesos. Por ello, el principal objetivo de este artículo es discutir un modelo con cierto impacto internacional, interesante y válido en el análisis de los procesos de aprendizaje; pero con algunas consideraciones culturales que hacen necesaria su redefinición.

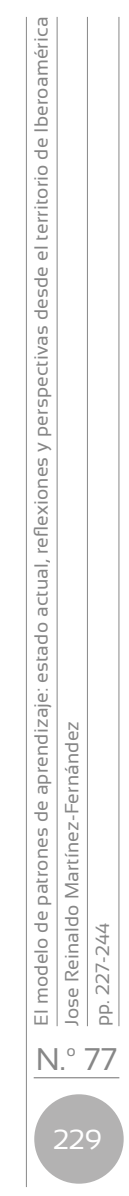




\section{Patrones de aprendizaje: el modelo y su historia}

En 1998 Jan D. Vermunt publica su artículo pionero en el que presenta el modelo de patrones de aprendizaje basado en un interés por la regulación de los procesos constructivos que inciden en los resultados de aprendizaje. El autor parte de la discusión acerca de dos visiones del aprendizaje, una que concibe al estudiante como un sujeto pasivo, consumidor de conocimiento y externamente dirigido (lo que luego será el patrón RD), en contraposición a la búsqueda de un sujeto que concibe el aprender como un proceso activo, constructivo y autodirigido (lo que luego será el patrón MD). En su interés por indagar acerca de estas dos concepciones de los procesos de aprendizaje, de entender por qué se dan tales procesos, realizó entrevistas en profundidad a estudiantes de cursos presenciales regulares y a estudiantes universitarios que seguían un programa a distancia.

Así, confirmó la presencia de cuatro componentes clave en la comprensión de los procesos de aprendizaje. Dos de ellos ampliamente estudiados hasta finales de la década de los noventa: las estrategias cognitivas y las motivacionales; y otros dos con menos tradición de investigación, como las estrategias de regulación y las concepciones de aprendizaje. A partir de ello, además, formuló que las estrategias de regulación están influidas por las concepciones de aprendizaje y en menor medida por la orientación motivacional. Además, ese nivel determinado de regulación es el componente central y mediador de las estrategias cognitivas.

Para el análisis de los componentes del modelo, Vermunt construye un instrumento (con 241 ítems) que denomina Inventory of Learning Style (ILS) con 50 ítems para cada uno de los componentes de procesamiento, regulación y orientación motivacional; y curiosamente plantea 91 ítems para las concepciones de aprendizaje, que en principio denominó modelos mentales. Las cuestiones referidas a las estrategias se responden en una escala tipo Likert de 1 a 5, desde "No lo hago nunca" hasta "Lo hago siempre"; mientras que los ítems de creencias se responden desde "Totalmente en desacuerdo" hasta "Totalmente de acuerdo", en una escala igual de 1 a 5. Luego, en una versión revisada del ILS, reducido a 144 ítems, el autor propone 30 ítems para cada componente de regulación, procesamiento y orientación motivacional, respectivamente; y para el componente de modelos mentales (concepciones de aprendizaje) plantea 54 ítems. Finalmente, durante el estudio de 1998, el autor redujo el ILS a su versión más conocida y aplicada de 120 ítems distribuidos en las subescalas de procesamiento (27 ítems), regulación (28), orientación motivacional (25) y concepciones de aprendizaje (40). En el tercer apartado se discutirá acerca de esta construcción tan amplia y no equivalente en la distribución de los ítems en cada componente y subescala. 
Cabe resaltar que cada componente contiene diferentes subescalas. Así, las estrategias de procesamiento se clasifican en profundo, paso a paso y concreto. La regulación, en autorregulación, regulación externa y ausencia de regulación. Las concepciones de aprendizaje, en construcción, bloques de información, uso del conocimiento, educación estimulada y el aprendizaje como necesariamente cooperativo. Finalmente, la orientación motivacional puede estar centrada en el interés personal intrínseco, los certificados, la autoevaluación, la vocación o la ambivalencia. En los diferentes artículos que conforman este dosier se presentan más detalles de cada una de ellas.

Con relación a la reducción de los ítems, el autor describe que se realizó con la finalidad de disponer de una versión del ILs utilizable (Vermunt, 1998). Además, se modificaron ligeramente los ítems para que fueran aplicables tanto en ambientes de aprendizaje presencial regular como en ambientes de aprendizaje universitario a distancia. El proceso de reducción se basó en la eliminación de ítems extremos, con medias muy altas o muy bajas, aquellos que tenían una desviación típica muy pequeña; y sobre la base de un análisis de componentes principales se suprimieron aquellos que tenían baja saturación en todos los factores o un peso equivalente en más de uno. Finalmente, algunas escalas se corrigieron en función del coeficiente alfa. Este es uno de los puntos sobre los que se reflexiona en el tercer apartado del artículo: ciertas limitaciones en el ILs por su extensión, por el desequilibrio en la distribución de los ítems y por su formulación a partir de un contexto europeo que parece limitar la discusión intercultural acerca de los patrones de aprendizaje.

Vermunt (1998), empleando un procedimiento de análisis basado en componentes principales con rotación oblicua, halló en ambos contextos de aprendizaje (regular y a distancia) la misma distribución de las subescalas en los cuatro factores (patrones) que se conocen: MD, RD, UD y AD. Además, confirmó los denominados vínculos direccionales de acuerdo con los cuales los componentes de creencias (concepciones y orientación motivacional) influyen en las estrategias de regulación, y estas últimas en las de procesamiento, aunque el impacto de la orientación motivacional resulta mucho más pequeño en las estrategias de procesamiento que el de las concepciones de aprendizaje.

En el análisis de las estrategias de regulación como componente central del modelo, el autor halla que estas explican todas las estrategias de procesamiento; sin embargo, las de regulación externa se relacionan negativamente con las estrategias de procesamiento profundo, en ambos ambientes de aprendizaje. Igualmente, el autor halla relación significativa positiva entre una concepción constructivista del aprendizaje y las 
estrategias de autorregulación; y significativa negativa entre una concepción reproductiva del aprendizaje y la autorregulación. Sin embargo, hoy (Martínez-Fernández y Vermunt, 2015) se conoce del papel significativo positivo de la regulación externa, en la activación tanto de la propia autorregulación como del procesamiento profundo, otro punto sobre el que se reflexiona en el tercer apartado.

En cuanto a las estrategias de regulación, Vermunt (1998) identifica que la ausencia de regulación está estrechamente vinculada con la orientación motivacional ambivalente. Además, señala que el incremento en la ausencia de regulación se relaciona significativa y positivamente con una concepción cooperativa y estimulada del aprendizaje (lo que se conoce hoy como un patrón UD). Así, se aprecia que la definición del patrón UD tiene que ver con estudiantes que carecen de habilidades para regular sus procesos de aprendizaje, y acuden a los otros significativos (compañeros o docentes) para que les inspiren sobre qué, por qué y cómo actuar, lo que en muchas ocasiones puede explicar la relación entre el patrón UD y el reproductivo (RD). En esta misma línea argumental, y diferenciando el estudio de los patrones con relación a los estilos de aprendizaje, el autor destaca que las formas de aprender van más allá de una consideración bipolar entre dependiente o independiente de campo; profundo o superficial; constructivo o reproductivo, y propone un esquema de cuatro patrones que en los años recientes se ha visto ampliado, enriquecido y revisado.

Finalmente, en su contribución pionera, Vermunt (1998) resalta que no se trata de un modelo "nuevo" acerca de los procesos de aprendizaje, sino de una integración de componentes donde la metacognición desempeña un papel central. Además, el autor destaca la identificación de una subescala que informa acerca de la ausencia de regulación, un aspecto que hasta la fecha no se había planteado en ningún otro modelo. Por otra parte, el autor señala que estos "estilos" de aprendizaje no son tan estables como se había planteado en otros modelos, aunque sí reconoce la mayor estabilidad de las concepciones de aprendizaje y de la orientación motivacional; mientras que las estrategias de procesamiento y de regulación resultan más adaptables y cambiantes en función del ambiente de aprendizaje. De allí la hipótesis de la modificabilidad de los patrones de aprendizaje, y el cambio en la etiqueta del constructo, de estilos a patrones de aprendizaje.

Así, se trata de un modelo integrador que surgió con un llamado al cambio en las teorías del diseño de instrucción para que fomentaran un papel más activo de los estudiantes, de modo que el control de los procesos de aprendizaje debe pasar de los docentes a ellos. Para este fin, no parece ser efectiva una instrucción directa, sino que se debe partir de 
una modificación de las concepciones de aprendizaje y de las estrategias de regulación para que estas, al ser autorreguladas, tengan un efecto de acción profunda en las estrategias de procesamiento y, por ende, en los resultados de aprendizaje.

En el 2004, casi una década después de la concepción del modelo (formulado en 1996), el autor lidera una primera revisión de este y su impacto (Vermunt y Vermetten, 2004). De esta manera, identifica que el ILS - el instrumento que permite identificar los patrones de aprendizajeha sido empleado básicamente en los Países Bajos, pero también en Bélgica, Chipre, Finlandia e Inglaterra, por lo que hace referencia a Europa. Además, se reportan estudios desde Estados Unidos, Indonesia y Sri Lanka; y en Latinoamérica (Argentina y Brasil). Lo interesante de todas estas aplicaciones es la consistencia del instrumento, su validez interna y su potencial en la identificación de los cuatro patrones clásicos inicialmente definidos (MD, AD, RD y UD) en muestras de estudiantes universitarios. Cabe señalar que en Finlandia no se identificó el patrón UD, lo que el autor atribuye a la estricta y refinada selección de estudiantes universitarios que se realiza allí.

Otros estudios, similares a lo que se reporta en Iberoamérica y que se revisan en el segundo apartado del artículo, señalan que no se identificó el patrón AD como un factor diferenciador, sino que las distintas subescalas que lo configuran teóricamente se diluyen en otros factores. Estos resultados se interpretan por el marcado carácter vocacional y orientado a la aplicación de muchos ambientes de aprendizaje en la universidad (Vermunt y Vermetten, 2004). De esta manera, el autor destaca el papel de la dimensión cultural y de otros factores contextuales en la explicación de la dominancia de ciertos patrones de aprendizaje, tales como las características del dominio específico, la percepción de la docencia, el nivel de estudios en la universidad, entre otros. Asimismo, se pone el acento en posibles explicaciones basadas en diferencias según el género y la edad, que apuntarían a razones del desarrollo, la hipótesis evolutiva que se ha mencionado.

Así, en el 2004, a partir de la revisión de estudios que aplican el ILS, Vermunt y Vermetten (2004) plantean dos hipótesis para explicar las diferencias en los patrones de aprendizaje: el contexto o ambiente de aprendizaje y el desarrollo evolutivo. En tal sentido, explican que la falta de diferenciación en cuanto a los componentes de aprendizaje que reporta Klatter (1995, citada por Vermunt y Vermetten, 2004) en estudiantes de secundaria tiene que ver con la edad de los sujetos (12 años). Sin embargo, otros estudios en el territorio de Iberoamérica hallan tales inconsistencias en muestras de estudiantes de primero de universidad (García-Ravidá, 2017; Martínez-Fernández y García-Ravidá, 2012; Martínez-Fernández

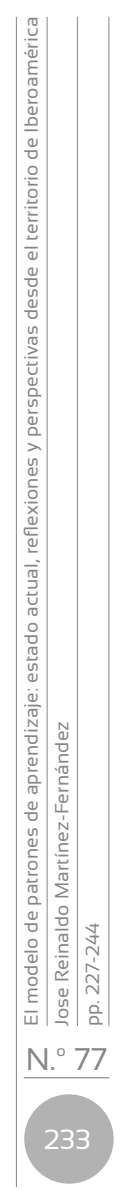


y Vermunt, 2015); y otros reportan una definición muy clara, coherente y consistente de los patrones de aprendizaje en el caso de muestras de estudiantes de Educación Primaria (Martínez-Fernández, Galera-Bassachs, y García-Orriols, 2017Quizás una explicación más apropiada sea una hipótesis referida a situaciones del contexto y de las etapas de cambio educativo o transición como factor clave en la inconsistencia o falta de diferenciación en la definición de los patrones de aprendizaje. La transición de una etapa educativa a otra, una institución educativa, el profesorado, e incluso cambios específicos de la edad en ciertos estudiantes podrían ser la clave para descifrar por qué, cuándo y cómo se modifican, o no, los patrones de aprendizaje. Así, parece que la hipótesis del contexto, o de la dimensión cultural del aprendizaje, es la que gana más fuerza frente a la hipótesis de la edad o del desarrollo.

Otro aspecto que se destaca en la revisión de Vermunt y Vermetten (2004) es que los componentes de los patrones de aprendizaje tienen cierta estabilidad y que el cambio es observable, pero no parece producirse en menos de un año. Además, la congruencia o consistencia entre las creencias y las acciones que Vermunt formuló en su modelo inicial no se observa en todas las muestras. Así, se identifican inconsistencias o disonancias como una concepción constructivista del aprendizaje relacionada con estrategias de procesamiento memorístico; o regulación externa explicando estrategias de procesamiento profundo. Lo que sí identifica es que ambos tipos de incongruencia o disonancias tienen un efecto negativo en los resultados de aprendizaje. Además, esta revisión da cuenta de algunos estudios con relación a los rasgos de la personalidad que demuestran que las puntuaciones en el patrón MD se relacionan con la apertura intelectual, el UD con el neocriticismo y el AD con la amabilidad. En cuanto al género, los estudiantes varones puntúan más alto en el patrón UD, y las mujeres en el RD. Estas tendencias son interesantes para tomarlas en cuenta en el diseño de acciones formativas.

En conjunto, hay una serie de estudios que demuestran la relevancia del modelo en cuanto a los componentes que identifica, pero con matices en función de una serie de variables personales y contextuales que el autor discute en otro artículo de la misma época (Vermunt, 2005). De este modo, los patrones de aprendizaje parecen una configuración que no siempre es consistente o consonante y que además se define a partir de un amplio rango de variables tales como la edad, el género, los conocimientos previos, las experiencias académicas, el dominio específico, los ambientes de aprendizaje, entre otras, que no permiten plantear un modelo explicativo claro acerca del cómo y el porqué de ciertos patrones 
de aprendizaje. Sin embargo, cabe destacar que a medida que los estudios se realizan en estudiantes universitarios avanzados, o de mayor edad, la consistencia de los componentes del modelo es más evidente.

Ahora bien, donde sí parece que surge una explicación algo más clara sobre el efecto de los patrones es cuando se hace referencia al rendimiento académico o los resultados de aprendizaje. Así, los estudios revisados (Vermunt y Vermetten, 2004) muestran que los mejores resultados de aprendizaje se relacionan con el patrón $\mathrm{MD}$, aunque en algunos casos también con el RD. El patrón AD parece el que menos efecto tiene en todas las direcciones, mientras que el UD, especialmente, pero también el RD se asocian a peores resultados académicos. Por último, en esta revisión se destaca la necesidad de diseñar acciones formativas que fomenten una concepción de aprendizaje constructiva y la autorregulación de los procesos de aprendizaje, pues parece que tal forma de instruir sí influye positivamente en la consolidación de un patrón MD O AD.

Para cerrar este recorrido histórico por el modelo de patrones de aprendizaje, y desde las revisiones lideradas por su autor, se comenta la discusión más reciente que se ha publicado (Vermunt y Donche, 2017). Se trata de una revisión de diversos estudios desde el 2004 hasta el 2016 en la cual se recoge una serie de datos que muestran la ampliación territorial en la discusión del modelo y de la utilización de su instrumento definitorio (el ıLs) alrededor del mundo. Además, se aprecia que se aborda no solo a los estudiantes universitarios de primer año de carrera, sino de diferentes niveles de secundaria, en formación de adultos, en formación profesional y hasta en el contexto de las empresas y de las organizaciones. Igualmente, se reporta un mayor número de estudios longitudinales en los que, también, se han diversificado e intensificado las mediciones estadísticas que se emplean; en suma, estudios metodológicamente más robustos. Para finalizar, se destaca que desde el 2012 hay cada vez más estudios que diseñan acciones formativas orientadas a fomentar los mejores componentes (MD y AD) de los patrones de aprendizaje.

En conclusión, y sobre la base de esta breve revisión histórica, se confirma la frecuente y clara identificación de los patrones inicialmente descritos ( $M D, A D, R D$ y UD). Asimismo, se confirma, en diferentes muestras alrededor del mundo, la estrecha y significativa relación del patrón MD con los mejores resultados de aprendizaje, y lo contrario en el caso de los patrones RD o UD. Por otro lado, se pone en evidencia que los patrones de aprendizaje están influidos por una amplia variedad de factores personales y contextuales que sugieren la necesidad de un modelo multicausal, espiral que tome en cuenta la personalización de los procesos de aprendizaje. 
Así, aspectos como la personalidad, la motivación académica, las metas de aprendizaje, las atribuciones causales, la autoeficacia, el esfuerzo, la edad, el género, los conocimientos previos, entre otros, plantean un complejo reto en el futuro de los marcos teóricos, metodológicos y prácticos a la hora de identificar, discutir y/o fomentar las "mejores" formas de aprender y enseñar. Además, parece obvio que el dominio específico, la estrategia de enseñanza y las percepciones personales que se tienen de los ambientes de aprendizaje son elementos clave para dibujar esas líneas de influencia, y sobre esa base inspirar el diseño de acciones formativas auténticamente transformadoras. Este es otro aspecto que se comentará en las perspectivas: la necesidad de diseñar itinerarios adaptativos y personalizados de aprendizaje.

\section{Patrones de aprendizaje en Iberoamérica: estado actual}

El estudio del modelo de Jan Vermunt en Iberoamérica se inicia a comienzos del siglo xxı (Alves de Lima et al., 2006). Sin embargo, es a partir de la traducción y adaptación de Martínez-Fernández et al. (2009) cuando se inicia una mayor indagación acerca del modelo en el territorio de lberoamérica y el debate en torno a él. De esta manera, se aportan datos a la discusión de los patrones de aprendizaje en otros contextos, cosa que recientemente Vermunt ha señalado como relevante y necesaria (Vermunt y Donche, 2017).

Una consulta en la base de datos Scopus@ de las palabras clave patrones de aprendizaje (en castellano) y Vermunt, en todos los campos, arroja un resultado de solo ocho artículos (17 de mayo del 2009). De ellos, cinco emplean el modelo de Vermunt y el instrumento ILS, los otros tres únicamente lo citan. A continuación, un breve resumen y comentarios acerca de seis de estos artículos que se consideran de interés para la investigación acerca de los patrones de aprendizaje en Iberoamérica.

En orden cronológico, el primer estudio lo firma Vázquez (2009), quien reporta la identificación de los cuatro patrones de aprendizaje $(\mathrm{MD}$, $A D, R D$ y UD) en una muestra de 420 estudiantes de primer año de Ingeniería de Buenos Aires (Argentina). En su estudio emplea una versión del ILS de 100 ítems y concluye que predomina el patrón AD (concepción de aprendizaje basada en el uso del conocimiento, orientación vocacional y procesamiento concreto). Sin embargo, destaca el bajo índice en las subescalas de autorregulación. En cuanto al rendimiento académico halla relaciones diferenciadas en función del tipo de asignatura o dominio específico. Por ejemplo, las estrategias de memorización se relacionan de 
modo significativo positivo con la calificación en la asignatura de análisis matemático, pero ocurre todo lo contrario (relación significativa negativa) con la calificación en química. Coincidimos con la autora en que seguramente los estudiantes adaptan sus estrategias de procesamiento al estilo de enseñanza, apreciación que coincide con los planteamientos de Vermunt (1998) acerca de la mayor variabilidad en las estrategias, no en las concepciones o motivos para aprender. En todo caso, se confirma el papel significativo positivo del patrón MD y todo lo contrario en el caso del RD.

Le sigue el artículo de Martínez-Fernández y García-Ravidá (2012), quienes analizan una muestra de 101 estudiantes del Máster de Profesorado en Educación Secundaria de la Universidad de Granada (España). Para ello utilizan la versión del ıs en español de Martínez-Fernández et al. (2009) y un cuestionario de datos personales que indaga acerca de variables personales y contextuales. Los autores no replican los patrones de Vermunt y lo explican por una posible hipótesis fundamentada en razones culturales, en el territorio de lberoamérica. Así, se identifica un patrón MD con regulación externa, un patrón pasivo basado solo en creencias y motivaciones, un patrón RD/UD y se aíslan las subescalas de orientación a las calificaciones. En cuanto al resto de variables analizadas destaca la puntuación más alta de las mujeres en el patrón pasivo, y que a mayor mayor edad, paradójicamente, mayor puntuación en las subescalas del patrón reproductivo (RD). Por último, un asunto relevante es que los autores identifican que el esfuerzo reportado por los estudiantes tiene un papel significativo positivo en su rendimiento académico; sin embargo, señalan que ese esfuerzo se combina de manera diferenciada y personalizada con ciertos indicadores de los patrones de aprendizaje. En próximos apartados se comentan tres vías explicativas que reportan Martínez-Fernández y Vermunt (2015) en el camino de la autorregulación al éxito académico.

En tercer lugar, y en línea con la necesidad de ampliar el estudio de los patrones de aprendizaje en otros territorios, se halla el capítulo en la colección New Perspectives on Learning and Instruction de Vermunt et al. (2014). En particular, los autores indican que el creciente intercambio internacional de estudiantes, profesores y responsables político-administrativos de los sistemas de Educación Superior requiere una amplia comprensión de las formas de aprender, y agregamos: de ser y de vivir, en otros contextos. Así, la comprensión de las diferentes vías de éxito o fracaso académico deben contribuir a mejorar los procesos de adaptación a otras culturas de aprendizaje, pero desde el enriquecimiento mutuo, en tono de inclusión. Los autores parten del estudio de Marambe, Vermunt y Boshuizen (2012) en el cual se comparan las puntuaciones del ıLS entre dos países asiáticos (Indonesia y Sri Lanka) y Holanda, e incluyen China 
entre los asiáticos; y por Iberoamérica se incluyen datos provenientes de Colombia, España, México y Venezuela. En una muestra total de 3855 estudiantes universitarios, los autores identifican alta consistencia interna del ILS, a excepción del componente de orientación motivacional. Este resultado remite a Vermunt y Vermetten (2004), cuando exponían el bajo impacto del componente de orientación motivacional dentro del modelo. Además, confirman datos de otros estudios que señalan la subescala de interés personal como la más débil de todas. Los análisis revelan diferencias significativas, entre todas las muestras, en todas las subescalas, salvo en la regulación externa y el procesamiento paso a paso. De ahí se puede plantear la hipótesis de actuación similar en lo que hace a la regulación externa y el procesamiento superficial en variados contextos culturales.

En cuanto a las diferencias, los estudiantes colombianos reportan mayor puntuación en las subescalas del patrón MD, pero también en la regulación externa o en la ausencia de regulación. Los estudiantes de Venezuela presentan las mayores puntuaciones en la regulación externa, los de España en la necesidad de estimulación docente y los de Indonesia en el patrón RD. Así, los autores señalan diferencias entre continentes pero también entre países de un mismo continente. Por ello afirman $-\mathrm{y}$ estamos de acuerdo- que los patrones se observan alrededor del mundo, y un aspecto clave es analizar los aspectos más individuales y personalizadores de las formas de aprender, como ya se ha puntualizado.

Quizás lo que se diferencia a través de los continentes es una cultura o estilo de enseñanza que influye en la configuración de los patrones de aprendizaje. Sin embargo, tal influencia estará mediada por indicadores personales y percepciones muy particulares de los ambientes de aprendizaje. Esta es una razón por la que algunos estudiantes asiáticos están más cómodos en una universidad europea, pero otros no, o por la que estudiantes españoles se adaptan mejor que otros a una universidad de Latinoamérica. De alguna manera, la persona encuentra su espacio más allá de sus fronteras, se enriquece y crece; pero otros (estudiantes y profesores) sufren un efecto negativo de la fricción que genera la transición. Esto cuestiona la hipótesis de la dimensión cultural con respecto al aprendizaje, y pone la mirada en los modelos y estrategias y de enseñanza; pero además en cómo los percibe, vive y gestiona, o no, la persona en proceso de aprendizaje.

Vermunt et al. (2014) apuntan aspectos muy interesantes en relación con los patrones de aprendizaje. Así, señalan la identificación de los cuatro patrones en diferentes territorios, destacan la consistencia del patrón MD, pero también de la presencia de la regulación externa en conjunto con indicadores de autorregulación, aspecto contradictorio en los orígenes del modelo. Los patrones pasivos son otro de los puntos de interés de 
esta revisión: por un lado el pasivo-idealista que combina las creencias de aprendizaje, y por otro el pasivo-motivacional que reúne los motivos para aprender; en ninguno de estos casos hay actividad o estrategia alguna que les corresponda. En suma, parece claro que el modelo, y otras aproximaciones acerca de los procesos de enseñanza y aprendizaje requieren de datos internacionales y diversas miradas que permitan validar hipótesis, discutir visiones y enriquecer el conocimiento científico sobre ellos.

El cuarto artículo que se identifica es una contribución de Cela-Ranilla y Gisbert (2013), quienes analizan una muestra de 699 estudiantes de primer año de carrera de varias titulaciones de una universidad española. Sin embargo, aunque utilizan el término patrones de aprendizaje, los autores se apoyan en el modelo de aprendizaje interactivo (ILM, por sus siglas en inglés) de Johnson, y no en la propuesta de Vermunt, que es citado en el artículo. Aun así se destaca que los autores encuentran que los estudiantes, en general, siguen un tipo de patrón reproductivo (RD) y de alta dependencia en la regulación externa.

Rosário et al. (2014) firman la contribución número cinco, que consiste en una revisión sistemática del 2001 al 2011 acerca del constructo Autorregulación del aprendizaje; pero solo refieren el artículo de Vázquez (2009) por su utilización del modelo de Vermunt (1998).

Finalmente, Martínez-Fernández y Vermunt (2015) firman quizás el artículo más amplio que conocemos acerca de los patrones de aprendizaje, a la fecha, en estos ranking de impacto, por ejemplo Scopus $\bigcirc$. En este artículo, se profundiza en el análisis de la muestra de 456 estudiantes iberoamericanos que formaron parte de la revisión de Vermunt et al. (2104). Los autores destacan la consistencia interna del ILS en español (Martínez-Fernández et al., 2009), a excepción de la subescala de interés personal, como ya se ha reportado en otros estudios. Los autores van un poco más allá y plantean como hipótesis que aspectos como "enriquecimiento intelectual" o "disfrute, relax", no son precisamente las sensaciones u orientación motivacional de los estudiantes universitarios en Iberoamérica. En este territorio, por lo general, alcanzar niveles educativos de educación superior es un desafío personal e incluso familiar, y ello implica presión social, altos niveles de estrés académico y ansiedad. Así, parece claro que se debe revisar la subescala de motivación intrínseca; e incluso se debe redefinir todo el componente de orientación motivacional, como se aprecia en estudios con muestras de otros territorios fuera de Europa (Marambe et al., 2012). Además, los autores destacan una vez más el papel relevante de las estrategias de regulación externa, las cuales tienen un importante peso explicativo sobre la autorregulación definiendo la paradoja española y latinoamericana. 
Con relación al rendimiento académico, se encontró que el esfuerzo es una variable clave en cuanto a su combinación con los patrones de aprendizaje, en línea con Martínez-Fernández y García-Ravidá (2012). Finalmente, el resultado que nos parece más interesante y que seguramente resume mucho de lo hallado en Iberoamérica, en cuanto al modelo de patrones de aprendizaje, es la definición de tres vías explicativas para el desempeño académico: (1) activación de las estrategias de procesamiento profundo a partir de las estrategias de regulación externa y de una concepción reproductiva del aprendizaje (incongruencia positiva); (2) activación del procesamiento profundo desde una concepción constructivista del aprendizaje y con regulación externa (la paradoja iberoamericana); (3) y la vía teórica, y práctica, conocida de la activación del procesamiento profundo desde una concepción constructivista mediada por la autorregulación (consonancia compleja). Esta última vía, además, se puede identificar con o sin un esfuerzo significativo por parte del estudiante universitario; y ese esfuerzo tiene una vía explicativa diferenciada (Martínez-Fernández y Vermunt, 2015).

Por lo expuesto, se aprecia que el modelo de patrones de aprendizaje aporta una consistente visión de los procesos de aprendizaje. Sin embargo, su expansión y discusión en Iberoamérica aún es escasa, o requiere de producciones de mayor calidad que lleguen a las bases de datos de impacto internacional. Se conoce de varios autores que han generado conocimiento en esta área, pero que los resultados de sus estudios no están en la mira de alcance que se eligió en esta revisión: la base Scopus@ . Por otro lado, aquí se ha centrado la revisión en estudiantes universitarios, pero se sabe que comienzan a surgir estudios, desde Iberoamérica, que analizan los patrones de aprendizaje en Educación Primaria y en el contexto de las organizaciones (aprendizaje no formal).

\section{Patrones de aprendizaje: potencialidades, limitaciones y perspectivas}

La revisión realizada en este artículo, sobre la historia reciente del modelo de patrones de aprendizaje (Vermunt, 1998) y su alcance en Iberoamérica genera un conjunto de reflexiones acerca de las potencialidades, limitaciones y perspectivas en esta área, las cuales se listan a continuación. En primer lugar, tres potencialidades que apuntan a la fuerza del modelo; y luego seis limitaciones y sus correspondientes perspectivas. 


\section{Potencialidades}

» La definición de 20 subescalas (o 16, según como sean consideradas) que reflejan altos índices de consistencia interna en diversas muestras de universitarios alrededor del mundo. Además, los hallazgos en diferentes territorios y las relaciones reportadas con respecto al rendimiento académico indican un alto índice de validez externa y concurrente con otras variables según el tipo de patrón de aprendizaje.

» La existencia de cuatro patrones ampliamente reconocidos $(\mathrm{MD}, \mathrm{AD}$, RD y UD), así como de dos patrones pasivos (idealista y motivacional) y de un patrón flexible, datos que son reportados sistemáticamente en diferentes tipos de muestras poblacionales, dominios específicos o niveles educativos.

» La promulgación y confirmación de unos vínculos direccionales que permiten considerar el peso predictivo de las concepciones de aprendizaje y de la orientación motivacional (en menor escala) sobre las estrategias de procesamiento y de regulación.

\section{Limitaciones}

» Se basa en un instrumento (el ILS) que parece extenso (120 ítems), concebido en el seno de grupos de investigación europeos. A la fecha, se conoce una nueva versión reducida (60 ítems) y equilibrada con respecto a los componentes (15 ítems cada uno) y las subescalas (3 ítems en cada una de las 20 subescalas) (Martínez-Fernández y García-Orriols, 2017).

» El modelo pone el peso central en las estrategias de autorregulación, pero se ha demostrado que en diferentes territorios, especialmente en Iberoamérica, la regulación externa tiene un alto valor en la configuración de los procesos de aprendizaje, e incluso en la explicación paradójica del éxito académico. En consecuencia, modelos explicativos de los procesos de aprendizaje con aspiración internacional e inclusiva del desarrollo científico requieren la consideración de tal aspecto.

» La mayoría de estudios se han centrado en los primeros años de la universidad; si bien dibujan claramente las etapas de fricción asociadas al acceso a otros niveles educativos, no se conoce cuánto dura tal fricción ni sus efectos, dado que son escasos los estudios longitudinales en esta área del conocimiento. 
» Se presenta un modelo y un conjunto de estudios centrados en el análisis de las variables que lo conforman. Así, se ofrece una mirada general, global y de tendencias grupales que deja a un lado la personalización de los procesos de aprendizaje.

» Ciertamente, se trata de un modelo potente de los procesos de aprendizaje pero con débil impacto en la explicación del rendimiento académico. Quizás una vía indirecta para el cuestionamiento de los procesos de evaluación de los aprendizajes en la universidad.

»Son escasos los estudios que reportan datos sobre la intervención educativa basada en el modelo de los patrones de aprendizaje. ¿Qué se puede decir a las estrategias de enseñanza a partir de los resultados sobre los patrones de aprendizaje?

\section{Perspectivas}

» Desarrollar material metodológico instrumental que permita un enfoque intercultural de los diferentes componentes de los patrones de aprendizaje.

» Se hace necesario desarrollar vías explicativas acerca del papel de la regulación externa en la configuración de los procesos de aprendizaje.

» Se requiere un mayor número de estudios que aborden los niveles educativos previos y posteriores a la universidad, en la medida de lo posible de corte longitudinal. Así, Educación Primaria y aprendizaje en las organizaciones son dos de los ámbitos que es preciso indagar para discutir la validez del modelo más allá de lo que se conoce de los estudiantes universitarios.

» Diseñar estudios centrados en las personas que nos reporten datos de perfiles individualizados, de conglomerados, y de una mirada más centrada en los sujetos. Diseñar y discutir el impacto de itinerarios adaptativos personalizados en los patrones de aprendizaje y en el rendimiento académico.

» Necesidad de estudios que aborden el impacto específico de las diferentes subescalas que mide el ILS, en el rendimiento académico, en un análisis más detallado y específico que profundice en los resultados que se basan en la puntuación general del patrón de aprendizaje.

» Avanzar en el diseño de acciones formativas y/o modelos de enseñanza basados en lo que se conoce acerca de la identificación y el impacto de los patrones de aprendizaje. 
Para concluir, y volviendo a la metáfora de la navegación que introduce a este dosier, los patrones de aprendizaje tratan de un modelo acerca de qué, por qué y cómo aprender, de claro anclaje en una visión eurocentrista, pero que en su corta historia ha tenido la fuerza de penetrar y validarse en "otras aguas". En el caso del territorio de Iberoamérica, un navegar tímido pero que cala y moja. Un conocimiento poco expandido y por ende poco o nada discutido en las revisiones que se han realizado del modelo. Así, una nave potente, que requiere ajustes (como todo proceso vital) pero que promete interesantes travesías si asimos el timón con fuerza y esfuerzo.

\section{Referencias}

Alves de Lima, A., Bettati, M. I., Baratta, S., Falconi, M., Sokn, F., Galli, A., Barrero, C., Cagide, A. e Iglesias, R. (2006). Learning strategies used by cardiology residents: Assessment of learning styles and their correlations. Education for Health, 19(3), 289-297. DOI: 10.1080/13576280600937788.

Cela-Ranilla, J. M., y Gisbert, M. (2013). Patrones de aprendizaje de los estudiantes universitarios de primer año. Revista de Educación, 361, 171-195.

García-Ravidá, L. B. (2017). Patrones de aprendizaje en universitarios latinoamericanos: dimensión cultural e implicaciones educativas (tesis doctoral). Universitat Autònoma de Barcelona, Barcelona, España.

Marambe, K. N., Vermunt, J. D., y Boshuizen, H. (2012). A cross-cultural comparison of student learning patterns in higher education. Higher Education, 64, 299-316. Dol: 10.1007/s10734-011-9494-z.

Martínez-Fernández, J. R., Galera-Bassachs, A. y García-Orriols, J. (2017). Patrones de aprendizaje en Educación Primaria: identificación y acciones formativas inclusivas. Barcelona: Wolters Kluwer:

Martínez-Fernández, J. R., García-Ravidá, L. B., González Velázquez, L., Gutiérrez-Braojos, C., Poggioli, L., Ramírez Otálvaro, P. y Telleria, M. B. (2009). Spanish version of the ILS. DOI:10.13140/ RG.2.2.13975.27048.

Martínez-Fernández, J. R. y García-Orriols, J. (2017). ıP short-version. Grup de Recerca Pafiu. Barcelona: Universitat Autònoma de Barcelona. DOI: 10.13140/RG.2.2.36074.00961.

Martínez-Fernández, J. R. y García-Ravidá, L. B. (2012). Patrones de aprendizaje en estudiantes universitarios del Máster en Educación Secundaria: variables personales y contextuales relacionadas. Profesorado. Revista de Currículum y Formación del Profesorado, 16(1), 165-182. 
Martínez-Fernández, J. R. y Vermunt, J. D. (2015). A cross-cultural analysis of the patterns of learning and academic performance of Spanish and Latin-American undergraduates. Studies in Higher Education, 40(2), 278-295.

Rosário, P., Pereira, A., Högemann, J., Figueiredo, M., Núñez, J. C., Fernández, E. y Fuentes, S. (2014). Autorregulación del aprendizaje: una revisión sistemática en revistas de la base Scielo. Universitas Psychologica, 13(2), 781-797.

Vázquez, S. M. (2009). Rendimiento académico y patrones de aprendizaje en estudiantes de ingeniería. Ingeniería y Universidad, 13(1), 105-136.

Vermunt, J. D. (1998). The regulation of constructive learning processes. British Journal of Educational Psychology, 68, 149-171. DOI:10.1111/j.2044-8279.1998.tb01281.x.

Vermunt, J. D. (2005). Relations between student learning patterns, and personal and contextual factors and academic performance. Higher Education, 49, 205-234. DOI: 10.1007/s10734-004-6664-2.

Vermunt, J. D., Bronkhorst, L. H. y Martínez-Fernández, J. R. (2014). The dimensionality of student learning patterns in different cultures. En D. Gijbels, V. Donche, J. T. E. Richardson and J. D. Vermunt, Learning Patterns in Higher Education: Dimensions and Research Perspectives (pp. 33-55). Londres-Nueva York: Routledge.

Vermunt, J. D. y Donche, V. (2017). A learning patterns perspective on student learning in Higher Education: State of the art and moving forward. Educational Psychology Review, 29, 269-299. Dol: 10.1007/ s10648-017-9414-6.

Vermunt. J. D. y Vermetten, Y. J. (2004). Patterns in student learning: Relationships between learning strategies, conceptions of learning, and learning orientations. Educational Psychology Review, 16(4), 359-384. 